\title{
REA E BIBLIOTECAS: DISCUSSÕES SOBRE A IMPORTÂNCIA DA CLASSIFICAÇÃO E REPRESENTAÇÃO
}

\author{
OER AND LIBRARIES: DISCUSSIONS ON THE \\ IMPORTANCE OF CLASSIFICATION AND \\ REPRESENTATION
}

Dayanne da Silva Prudencioa Lyvia Rocha de Jesus Araujob

\begin{abstract}
RESUMO
Objetivo: Discutir as manifestações dos recursos educacionais abertos (REA) nas bibliotecas, apresentar o método SPECC e o padrão de metadados IEEE LOM Learning Object Metadata. Metodologia: Pesquisa de natureza exploratória e descritiva que utiliza pesquisa bibliográfica e abordagem qualitativa para a apresentação dos resultados. Resultados: Apresenta a importância dos processos de classificação e representação descritiva para a busca, acesso, uso e difusão dos recursos educacionais abertos. Conclusões: Conclui os processos de organização, classificação e representação de REA influenciam na recuperabilidade dos recursos nos catálogos virtuais e consequentemente na promoção, acesso e uso destes.
\end{abstract}

Descritores: Recurso Educacional Aberto. Recurso de Informação. Fonte de Informação. Learning Object Metadata.

\section{INTRODUÇÃO}

Os processos de ensino e aprendizagem são, no decorrer de cada época, manifestados por diferentes atividades e artefatos. Estes vão sendo influenciados pelas abordagens teóricas que sustentavam o campo científico, pelo conhecimento produzido, pelas práticas desenvolvidas, pelas tecnologias disponíveis e - não menos importante - pelo contexto político, histórico e social.

No âmbito educacional, a popularização das tecnologias da informação e

a Doutora em Ciência da Informação pelo IBICT/UFRJ. Professora do Departamento de Biblioteconomia da UNIRIO. Email: dayanne.prudencio@unirio.br

b Mestranda em Ciência da Informação pelo IBICT/UFRJ. Email: araujolyvia@ufrj.br 
da comunicação (TIC) alteraram significativamente a forma de aprender e ensinar. Na visão de Moraes (1997), a influência das TIC na Educação, aliada ao questionamento de compreender o mundo em partes e não de maneira integrada, foram os elementos fundamentais para a emergência de um novo paradigma educacional, ainda no começo do século XX.

Nesta perspectiva, novas orientações e estratégias são encaminhadas visando romper com as estruturas não integrativas do paradigma educacional de aprendizagem passiva, advindo do contexto da revolução industrial. Estimula-se, portanto, que o processo educacional seja colaborativo, criativo, com diferentes oportunidades de aprendizagem e que os envolvidos possam interagir, compartilhar e cocriar diferentes recursos, orientando, assim, uma aprendizagem significativa (AUSUBEL, 1968).

No contexto dessas transformações e orientando-se pela perspectiva que o ambiente digital oportuniza para a criação colaborativa, a gestão compartilhada e o compartilhamento em larga escala, surgem os recursos educacionais abertos (REA). O termo REA foi cunhado em 2002 no Fórum da UNESCO sobre Softwares Didáticos Abertos, sendo estabelecidos como:

Materiais de ensino, aprendizagem e investigação em quaisquer suportes, digitais ou outros, que se situem no domínio público ou que tenham sido divulgados sob licença aberta que permite acesso, uso, adaptação e redistribuição gratuitos por terceiros, mediante nenhuma restrição ou poucas restrições (UNESCO, 2012, online).

No Congresso Mundial sobre Recursos Educacionais Abertos (REA) de 2012, a UNESCO tratou de elucidar que não apenas os materiais digitais podem ser considerados REA. Ou seja, livros didáticos, artigos acadêmicos tutoriais, slides, recortes entre outros, desde que estejam licenciados de forma aberta, gratuita e permitindo o uso e adaptação, também podem se enquadrar na categoria.

Os REA constituem-se como parte essencial do movimento de educação aberta - um movimento educacional cuja gênese é o livre acesso a oportunidades de aprendizagem, em que diferentes instituições de distintas áreas do conhecimento desenvolvem ferramentas, recursos e iniciativas para a promoção da educação (SANTANA; ROSSINI; LUCA PRETTO, 2012). Além 
disso, relacionam-se com as propostas da Agenda 2030 para o Desenvolvimento Sustentável da UNESCO, principalmente no que tange à ODS4 - Educação de Qualidade e ODS10 - Redução de Desigualdades.

No bojo destas transformações sociais e educacionais, as bibliotecas e seus profissionais têm papel importante, quer seja adaptando e criando REA, quer seja atuando nos processos de gestão, licenciamento, compartilhamento, armazenamento, difusão e uso destes recursos. Nas fronteiras destes processos está a organização e representação destes recursos. É sobre esse aspecto que esta comunicação se desenvolve.

A questão que nos propomos ${ }^{3}$ a abordar é a que se segue: Como os processos de organização e tratamento da informação influenciam na promoção, acesso e uso de recursos educacionais abertos no contexto brasileiro?

Como objetivos geral e específicos da pesquisa estabelecemos:

- Discutir as diferentes manifestações de REA em bibliotecas;

- Apresentar o método SPECC;

- Apresentar o padrão IEEE LOM - Learning Object Metadata;

Tendo apresentado as perspectivas norteadoras, a questão de pesquisa e os objetivos, a seguir apresentamos nossos procedimentos metodológicos.

\section{PROCEDIMENTOS METODOLÓGICOS}

A pesquisa é exploratória-descritiva quanto ao seu objetivo-fim e utilizase de pesquisa bibliográfica para desenvolvimento de seu arcabouço teórico. Desenvolve-se a partir de análise dos dados e demonstração dos resultados com abordagem qualitativa.

A abordagem exploratória justifica-se haja vista que ainda há pouco conhecimento acumulado e sistematizado na produção científica brasileira da área de Ciência da Informação envolvendo a organização e representação da informação e do conhecimento nos REA.

O material utilizado na investigação foi do tipo bibliográfico, sendo obtido

\footnotetext{
${ }^{3}$ Significativa parte do texto desta comunicação foi apresentado como resumo expandido no $\mathrm{V}$ COAIC (2021).
} 
através de pesquisa nas seguintes fontes de informação: BRAPCI, SciELO e Web of Science. As fontes bibliográficas consultadas são, respectivamente, das seguintes áreas de domínio: Ciência da Informação, Interdisciplinar e Educação. Portanto, correlacionadas a investigação proposta.

Como critérios de inclusão, foram adotados estudos em português e inglês, publicados em periódicos indexados nas bases citadas. Como critérios de exclusão, foram utilizados: idiomas diferentes dos já mencionados; indexados em bases distintas das anunciadas; materiais que não fossem artigos revisados pelos pares; e estudos que não tratassem especificamente de atividades relacionadas ao escopo de pesquisa (organização e representação da informação e do conhecimento nos REA).

Lüdke e Andre descrevem que a abordagem qualitativa possui algumas características, a saber:
Pesquisa qualitativa tem 0 ambiente natural como sua fonte direta de dados e o pesquisador como seu principal instrumento; os dados coletados são predominantemente descritivos; a preocupação com o processo é muito maior do que com o produto; o significado que as pessoas dão às coisas e à sua vida são focos de atenção especial pelo pesquisador; a análise dos dados tende a seguir um processo indutivo (LUDKE; ANDRE, 1986, p. 38).

Neste sentido, nossa opção se justifica pelo fato de que a abordagem qualitativa foi adequada para compreender o objeto de estudo proposto nesta investigação, ou seja, compreender como a organização e tratamento da informação influenciam a promoção, acesso e uso de recursos educacionais abertos no contexto brasileiro.

\section{REA E BIBLIOTECAS: DISCUSSÕES SOBRE SUA CLASSIFICAÇÃO E FUNÇÃO}

Em 2017, ocorreu, na Eslovênia, o segundo Congresso Mundial de REA, organizado pela UNESCO. Neste evento, desenvolveu-se um plano de ação para maior promoção e utilização de REA entre todos os países do globo terrestre. O plano, denominado Ljubljana OER Action Plan 2017, apresentou orientações concisas para que pesquisadores, bibliotecários, docentes, 
orientadores educacionais, administradores de políticas educacionais, em variados níveis, pudessem desenvolver iniciativas locais de ordenamento para a criação e difusão destes recursos. Importante ressaltar que essa foi a primeira menção feita aos bibliotecários institucionalmente no movimento de promoção de REA. O plano de ação recomendava ações em cinco áreas estratégicas, a saber:

Desenvolvimento da capacidade de encontrar, reutilizar, criar e compartilhar REA nos seus usuários; Questões de cultura e de linguagem; Garantia de acesso igualitário e inclusivo aos REA; Desenvolvimento de modelos sustentáveis em sua elaboração; Desenvolvimento de ambientes cooperativos para 0 estabelecimento das políticas para a construção dos REA (LJUBLJANA action plan, 2017, p. 2, tradução nossa).

A partir das diretrizes estabelecidas no plano, especialmente no que tange à atuação das bibliotecas e seus profissionais, revela-se a necessidade de conhecimento técnico para gerenciar tais recursos, bem como de técnicas e padrões adequados para representação, difusão e recuperação destes recursos. Dessa forma, colocando-os efetivamente à disposição da comunidade usuária.

Kleymeer, Kleinman e Hanss (2010) afirmam que os bibliotecários podem ocupar papel central nesta demanda, haja vista suas compêtencias e habilidades de difusão informacional. Desta forma, podem e devem realizar promoção das iniciativas REA em suas organizações e em eventos científicos e fóruns apropriados, prestar assessoramento na avaliação do conteúdo e processo de licenciamento autoral, identificar fontes de conteúdo aberto para reuso, orientar docentes e discentes na elaboração e busca de REA, descrever, classificar e indexar estes recursos visando uma melhor recuperação no futuro, orientar sobre plataformas de publicação e melhores práticas, incentivar e promover os REA como fontes de informação confiável, tal qual ocorreu com a Wikipédia no começo dos anos 2010, entre outras atividades.

Hirst (2019) destaca a responsabilidade que os bibliotecários possuem neste cenário e que estes devem engajar-se em ações orientadas à adequada a organização e compartilhamento dos conhecimentos representados nestes recursos. Neste sentido, há um fio condutor que mantém a conexão entre a organização e representação e a difusão de REA, sempre a partir de uma cultura orientada ao compartilhamento, uso, alteração, combinação, reuso e 
redistribuição. Em outras palavras, busca-se a democratização dos conhecimentos, bem como a produção de novos.

A partir dos estudos de Evangelista (2018) e Prudencio; Bernardi; Biolchini (2020), Araújo (2020) defende que nas bibliotecas os REA se manifestam como fontes de informação, recursos incorporados às coleções e como produto de trabalho intelectual dos próprios bibliotecários. Sendo, portanto, este último uma forma de produção de conhecimento destes profissionais.

No que tange à manifestação como fontes de informação disponíveis nas e para as bibliotecas, Bezerra (2008) aponta que as bibliotecas inseridas em instituições de educação, do nível fundamental ao superior, devem operar como "laboratórios de autoaprendizagem", isto é, espaços dedicados a oferecer às comunidades acadêmicas serviços e fontes de informação que contribuam para o desenvolvimento da autonomia de seus usuários através do conhecimento.

Sobre esse aspecto, Araújo (2020), em pesquisa nos catálogos de 120 repositórios institucionais (RI) de instituições de ensino superior federal brasileiras, identificou que apenas $12,5 \%$, ou seja, $15 \mathrm{RI}$, possuem a categoria REA classificada como fonte de informação nos catálogos. Desta forma, em alguma medida a informação e o conhecimento produzido pelas instituições e seus colaboradores e apresentada sob o formato de REA não tem sido observada como fonte de informação por estas bibliotecas.

Ao não serem reconhecidos como fontes de informação, acabam por não ser incorporados aos acervos ou constituindo-se como recursos armazenados nas coleções destas unidades.

Por outro lado, quando incorporados, Prudencio, Bernardi e Biolchini (2020), indicam que comumente as bibliotecas os armazenam como coleções em repositórios temáticos. Contudo, os autores recordam a possibilidade do desenvolvimento de repositórios específicos denominados como Repositórios de Recursos Educacionais Abertos.

Essa perspectiva de classificação, embora adequada, não esgota as características descritivas e temáticas destes recursos e, por conseguinte, pouco contribui para os processos de seleção e organização destes recursos. Nessa linha, George Stachokas (2012) criou o SPECC, um método para auxiliar a 
tomada de decisão quanto à disponibilização e o acesso a recursos eletrônicos específicos que pudessem ser adequadamente gerenciados pela equipe de uma biblioteca ou unidade de informação e facilmente acessados por seus usuários.

O trabalho de Stachokas (2012) estabelece critérios de seleção, organização e inserção em coleções específicas. O modelo foi apresentado pela primeira vez na Conferência de Recursos Eletrônicos e Bibliotecas, em 2011, no Texas. A sigla SPECC é usada para definir os cinco critérios básicos de inclusão que norteiam o sistema, traduzidos como Conhecimento, Persistência, Entidade, Compatibilidade, e Conveniência, respectivamente.

O critério de Conhecimento se divide em quatro classes amplas: conhecimento revisado por pares, conhecimento sobre outro conhecimento, conhecimento técnico/comercial e conhecimento popular. A Persistência estima a longevidade de um recurso livre, por quanto tempo é garantida sua disponibilidade. A Entidade se refere à organização ou indivíduo que fornece o acesso, a plataforma ou mantém o site de domínio dos recursos. Esses três critérios são considerados universais, por serem aplicáveis de forma idêntica, independentemente do contexto.

Os outros dois, Compatibilidade e Conveniência, tratam sobre quão bem o recurso eletrônico funciona com as ferramentas de gerenciamento, pesquisa e descoberta da biblioteca e a familiaridade dos usuários com o idioma, a tecnologia, o dispositivo comumente usados e as características dos próprios recursos. Esses critérios são tidos como locais, já que variam em consonância com as políticas individuais das unidades em que são aplicados (STACHOKAS, 2012).

Cada critério do sistema de Stachokas utiliza o desdobramento em quatro classes para qualificar a profundidade dos materiais classificados. Essa disposição dos critérios e das classes está representada no quadro abaixo.

Quadro 1 - Relação dos critérios, classes e símbolos SPECC

\begin{tabular}{|c|l|c|}
\hline Critério & \multicolumn{1}{|c|}{ Classe } & Símbolo \\
\hline Conhecimento & - Revisado por pares & I \\
& - Sobre outro conhecimento & II \\
& - Técnico / Comercial; & III \\
& - Popular & IV \\
\hline
\end{tabular}




\begin{tabular}{|c|c|c|}
\hline Persistência & $\begin{array}{l}\text { - Altamente persistente (espera-se que dure mais } \\
\text { de } 10 \text { anos) } \\
\text { - Persistente (espera-se que dure mais de } 5 \\
\text { anos) } \\
\text { - Persistência incerta (incerta ou prevista para } \\
\text { durar menos de } 5 \text { anos) } \\
\text { - Temporária (conhecida por estar disponível } \\
\text { apenas por um breve período) }\end{array}$ & $\begin{array}{l}P+ \\
P= \\
P-= \\
T\end{array}$ \\
\hline Entidade & $\begin{array}{l}\text { - Formal (Uma organização formal) } \\
\text { - Informal (um grupo informal) } \\
\text { - Individual (Um site pessoal) } \\
\text { - Especial (um autor individual postando conteúdo } \\
\text { em uma plataforma mantida por uma entidade } \\
\text { maior). }\end{array}$ & $\begin{array}{l}\text { Ef } \\
\text { Ei } \\
\text { In } \\
S\end{array}$ \\
\hline \multicolumn{3}{|l|}{ Critérios Locais } \\
\hline Compatibilidade & $\begin{array}{l}\text { - Altamente Compatível } \\
\text { - Compatível } \\
\text { - Parcialmente Compatível } \\
\text { - Não Compatível }\end{array}$ & $\begin{array}{l}\text { C+ } \\
\text { C } \\
\text { C- } \\
\text { D }\end{array}$ \\
\hline Conveniência & $\begin{array}{l}\text { - Muito conveniente para os usuários } \\
\text { - Conveniente para os usuários } \\
\text { - Um pouco conveniente para os usuários } \\
\text { - Requer mediação da equipe para que os } \\
\text { usuários acessem o conteúdo }\end{array}$ & $\begin{array}{l}\mathrm{V}+ \\
\mathrm{V} \\
\mathrm{V}- \\
\mathrm{M}\end{array}$ \\
\hline
\end{tabular}

Fonte: STACHOKAS (2012 [tradução nossa])

*Observações: para bibliotecas com fundos limitados, os recursos eletrônicos gratuitos às vezes podem substituir assinaturas pagas, mas não se os recursos eletrônicos forem temporários ou improváveis de serem arquivados; os recursos eletrônicos da Classe Ef são considerados os mais responsáveis e confiáveis em termos de Entidade.

Ao classificar um REA conforme o sistema de Stachokas (2012), podemos conceber como mais adequados para aquisição: os objetos de aprendizagem revisados por pares, altamente persistentes, formais, altamente compatíveis e muito convenientes, descritos pela simbologia I, $\mathrm{P}+, \mathrm{Ef}, \mathrm{C}+, \mathrm{V}+$.

Certas tecnologias podem aperfeiçoar esse processo e tornar mais intuitivo o trabalho de bibliotecas, especialmente as que possuem contingente limitado de funcionários e um grande volume de REA e outros recursos eletrônicos para organizar. Dentre elas, há uma citada por Stachokas (2012) que aplica valores quantitativos aos critérios usados em seu esquema, o "Free Electronic Resource Classification Rating" de Paul Asay (2011), que converte as classificações qualitativas em uma escala numérica de 0 a 100, facilitando ao gestor a visualização estatística do material que é potencialmente mais acessível e mantido pela biblioteca. 
A classificação SPECC pode ajudar a decidir se os softwares de gerenciamento e armazenamento de coleções eletrônicas podem rastrear esses recursos usando somente os buscadores próprios da biblioteca ou se é mais apropriado estabelecer links entre bases para uma navegação generalizada dos usuários.

Portanto, o método de Stachokas (2012) permite uma um melhor gerenciamento das coleções de REA nas bibliotecas, permitindo que tais recursos sejam mais facilmente buscados, identificados e selecionados para ocupar uma coleção nos acervos, físicos ou digitais.

A respeito da manifestação como produção de conhecimento dos bibliotecários, temos nos estudo de Kleymeer, Kleinman e Hanss (2010) e Prudencio, Bernardi e Biolchini (2020) importantes contribuições. Há indicações de iniciativas internacionais como a SPARC (Editora Acadêmica e Coalizão de Recursos Acadêmicos) que, desde 2013, desenvolve ações que possibilitam o compartilhamento aberto de produtos de pesquisa e materiais educacionais, visando democratizar o acesso ao conhecimento, propiciar inovação e aumentar o retorno sobre nosso investimento em pesquisa e educação.

Também destacamos a Certificação em Biblioteconomia de REA promovida pela Open Textbook Network em parceria com a The Association of College and Research Libraries (ACRL), uma divisão da American Library Association.

Neste programa, os bibliotecários recebem treinamento formal aplicado a partir de mentores especialistas para construir programas de educação aberta sustentáveis, colaborativos e eficazes em suas unidades de informação. Alguns dos tópicos deste programa são design instrucional, preservação digital, tecnologia para interfaces e licenças creative commons e direito autoral.

No cenário brasileiro, as iniciativas de REA envolvendo a participação de bibliotecários ainda são incipientes e sobretudo ocorrem na Fundação Oswaldo Cruz, onde um grupo de bibliotecários depositam os recursos educacionais, quase sempre resultado de cursos de qualificação onde atuam como docentes, no repositório institucional denominado ARCA. 
Discutir como estes recursos educacionais se manifestam nas bibliotecas permite que o profissional da informação desenvolva parâmetros adequados e necessários para organizar o conhecimento dispostos nestes recursos nos sistemas de informação, atividade fundamental para o processamento e recuperação de documentos/informação.

Outrossim, a atividade de representação destes recursos é fundamental. A adequada descrição destes objetos educacionais visa em algum nível aperfeiçoar, agilizar o processo de busca, promover uma melhor localização, acesso e de recuperação destes itens.

Para tanto, cada objeto informacional aciona o conjunto específico de metadados de seu contexto e pertinência. No Brasil, a maioria das UI vale-se do padrão Dublin Core (DC) para representação descritiva de seus itens. Contudo, para descrição de objetos educacionais recomenda-se a utilização do padrão IEEE LOM - Learning Object Metadata. Trata-se de padrão desenvolvido em 2002 pelo Learning Technology Standards Comitee (IEEE), objetivando estabelecer um conjunto mínimo de atributos para uma eficiente descrição de recursos educacionais, e ainda que vocabulários podem ser usados para tais descrições.

[Portanto] o padrão LOM especifica um cabeçalho que fornece informações sobre o objeto de aprendizagem. Os elementos que compõem esse cabeçalho são os metadados que descrevem semanticamente o objeto de aprendizagem. Assim, esse padrão não interfere no conteúdo ou nas regras dos objetos de aprendizagem, uma vez que apenas agrupa metadados (PÖTTKER; FERNEDA; MOREIRO-GONZÁLEZ, 2016, p. 31).

Segundo a IEEE LTSC (2002 apud PÖTTKER; FERNEDA; MOREIROGONZÁLEZ, 2016, p. 26), "a estrutura básica do padrão IEEE LOM para descrição de objetos de aprendizagem ocorre através de campos que estão agrupados por categorias e que permitem sua posterior recuperação". Neste sentido, o grande foco da descrição é apoiar a reutilização de objetos educacionais, auxiliar sua descoberta e facilitar a interoperabilidade, sobretudo no contexto de sistemas de gerenciamento de aprendizado online, tal como os repositórios e os Massive Open Online Courses (MOOC).

A seguir apresentamos a estrutura básica do padrão de metadados educacional LOM. 
Figura 1 - Categorias de metadados do padrão LOM

\begin{tabular}{|c|c|c|}
\hline 1. General & 2. Lifecycle & 3. Meta metadata \\
\hline 1.1 Identifier & 2.1. Version & 3.1. Identifier \\
\hline 1.2. Title & 2.2. Status & 3.2. Catalog \\
\hline 1.3. Catalog Entry & 2.3. Contribute & 3.2.1. Catalog \\
\hline 1.3.1 Catalog & 2.3.1. Role & 3.2.2 Entry \\
\hline 1.3.2. Entry & 2.3.2. Entity & 3.3. Contribute \\
\hline 1.4 Language & 2.3.3 Date & 3.3.1 Role \\
\hline 1.5.Description & & 3.3.2. Entityt \\
\hline 1.6. Keywords & & 3.3.3. Date \\
\hline 1.7. Coverage & & 3.4. Metadata Squema \\
\hline 1.8. Structure & & 3.5 Language \\
\hline \multicolumn{3}{|l|}{ 1.9 Aggregation level } \\
\hline 4. Technical & 5. Educational & 6. Righths \\
\hline 4.1. Format & 5.1. Interactivity Type & 6.1. Cost \\
\hline 4.2. Size & 5.2. Learning Resource Type & $\begin{array}{l}6.2 \text {. Copyright and others } \\
\text { restrictions }\end{array}$ \\
\hline 4.3 Location & 5.3. Interactivity level & 6.3. Description \\
\hline 4.4. requirements & 5.4. Semantic density & \\
\hline 4.4.1 Type & 5.5. Intended End User Role & \\
\hline 4.4.2 Name & 5.6. Context & \\
\hline 4.4.3. Minimum Version & 5.7. Typical Age Range & \\
\hline 4.4.4 Maximum version & 5.8. Difficulty & \\
\hline 4.5. Instalattion Remarks & 5.9. Typical Learning Time & \\
\hline $\begin{array}{l}\text { 4.6. Other Plataform } \\
\text { requirements }\end{array}$ & 5.10. Description & \\
\hline 4.7 Duration & 5.11. Language & \\
\hline 7. Relation & 8. Annotation & 9. Classification \\
\hline 7.1 Kind & 8.1 Person & 9.1. Purpose \\
\hline 7.2. Resource & 8.2. Description & 9.2. Taxon Path \\
\hline 7.2.1. Identifier & 8.3. Date & 9.2 .1 Source \\
\hline 7.2.2. Description & & 9.2.2 Taxon \\
\hline \multirow[t]{4}{*}{ 7.2.3. catalog Entry } & & 9.2.2.1. Id \\
\hline & & 9.2.2.2. Entry \\
\hline & & 9.3 Description \\
\hline & & 9.4 Keywords \\
\hline
\end{tabular}

Fone: Dutra (2003, p. 95)

Merece destaque e discussão os atributos 5 (Educationa), que agrupa as características educacionais e pedagógicas do recurso educacional; e 6 (Rights), que orienta os direitos de propriedade intelectual e condições de uso do objeto educacional (DUTRA, 2003).

Sobre este tópico, devemos esclarecer a importância do uso de licenças abertas, haja vista que na gênese dos REA estão 4 diretrizes, baseadas nos postulados das licenças Creative Commons, a saber: Reusar, ou seja, deve haver a liberdade de usar o original ou desenvolver uma nova versão com base neste; Aprimorar, que envolve a liberdade de efetuar alterações visando adaptação e melhoria no recurso educacional de modo a atender as necessidades do utente; Recombinar, que envolve combinar outros recursos e fazer misturas com outros REA para o desenvolvimento de novos materiais; e Distribuir, que envolve ter autorização para fazer cópias e compartilhar os 
recursos educacionais originais que você e outros criaram (UNESCO, 2015, grifo nosso).

Cumpre informar que, via de regra, todos os REA devem ser licenciados em creative commons, não devendo jamais serem atribuídas licenças de restrições como ND (Não-a-obras-Derivadas).

Em síntese, a adequada descrição dos REA, aqui incluindo-se o tipo de licenciamento empregado, é fundamental para uma infraestrutura informacional (pesquisa, edição, compartilhamento, download) satisfatória e consequentemente garante interoperabilidade, atendimento as necessidades dos utentes e promoção dos REA.

Retomando o aspecto dos atributos, o item 7 (Relation), também merece destaque ao agrupar características que definem a relação entre o objeto educacional e outros objetos educacionais correlacionados (DUTRA, 2003). Desta forma, este atributo permite ao bibliotecário identificar itens correlacionados na coleção. Outrossim, permite ao usuário identificar itens relacionados capazes de alterar o delineamento de estratégia pedagógica que se pretende implantar com a utilização de tais recursos.

Já o atributo 8 (Annotation) prevê comentários sobre o uso educacional do objeto e provê informação de quando e por quem foi ciado (DUTRA, 2003). Desta forma, contribui com notas pedagógicas para que bibliotecários e usuários saibam, por exemplo, a série de ensino, disciplinas aplicáveis ao recurso e questões para discussão com o recurso.

Portanto, verifica-se que o padrão IEEE LOM, ao colocar ênfase sobre os recursos educacionais, os especifica e, consequentemente, melhora a orientação à descrição dos objetos de aprendizagem.

Colocadas tais questões, reforça-se a ideia de que uma adequada organização, tratamento e descrição de recursos educacionais cooperam para uma melhor localização, acesso, avaliação e utilização destes. Há, assim, uma responsabilidade e papel a ser ocupado pelas bibliotecas e seus profissionais.

Através de suas práticas informacionais, as bibliotecas podem desempenhar um papel central na transformação do ensino e da aprendizagem, apoiando a adoção de REA. Ao mesmo tempo, conectando educadores, 
educandos e recursos da maneira mais eficaz possível, instruindo-os a pesquisar estes recursos, licenciá-los, utilizá-los e desenvolver novos.

\section{CONSIDERAÇÕES FINAIS}

Considera-se que todos os objetivos de pesquisa foram alcançados. Outrossim, a apresentação da aplicabilidade do método SPECC e o reforço sobre a importância da descrição dos REA suscitou respostas à questão de pesquisa.

Ainda é pouca a produção científica brasileira que trate das relações entre REA, bibliotecas e bibliotecários. Assim sendo, aponta-se para a necessidade de estudos mais aprofundados sobre os aspectos relacionados à organização $\mathrm{e}$ representação do conhecimento em e para REA. Igualmente, é importante que nas práticas de ensino de metadados, outros padrões, para além do Dublin Core, sejam apresentados aos futuros profissionais.

Entende-se que, ao apresentar o método SPECC de Stachokas (2012), contribuímos com a socialização de saberes sobre o gerenciamento de REA em bibliotecas e, empiricamente, oferecemos alternativas ao desenvolvimento de práticas biblioteconômicas apoiadas em conhecimento sistematizado.

Compreende-se a necessidade de maior envolvimento dos bibliotecários brasileiros no movimento da educação aberta e na promoção de REA. Além disso, aqueles que já possuem conhecimentos e práticas acerca de produção, tratamento, armazenamento e disseminação de REA devem socializar seus saberes e práticas contribuindo teórica e empiricamente para o desenvolvimento do campo.

\section{REFERÊNCIAS}

ARAUJO, L. R. J. REA em saúde: boas práticas para sua aplicação e o papel das bibliotecas. 2020. Trabalho de Conclusão de Curso (Graduação em Biblioteconomia) - Escola de Biblioteconomia, Universidade Federal do Estado do Rio de Janeiro, Rio de Janeiro, 2020.

ASAY, P. Free electronic resource classification rating. Cunningham memorial library, Indiana, 2011. Disponível em: 
http://library.indstate.edu/tools/freeschema/rating.html. Acesso em: 11 nov. 2019.

AUSUBEL, D. P. Educational psychology: a cognitive view. New York: Holt, Rinehart and Winston, 1968.

BEZERRA, M. A. C. O papel da biblioteca escolar: importância do setor no contexto educacional. CRB8 Digital, v. 1, n. 2, 2008. Disponível em: http://hdl.handle.net/20.500.11959/brapci/9177. Acesso em: 5 ago. 2020.

DUTRA, D. Uma arquitetura de biblioteca digital de Aulas baseada no padrão IEE LOOM. 2003. 104 f. Dissertação (Mestrado em Ciência da Computação) Universidade Federal de Santa Catarina, Florianópolis, 2003. Disponível em: http://www.inf.ufsc.br/ mario.dantas/material.pdf. Acesso em: 1 out. 2021.

EVANGELISTA, C. C. D. Recursos Educacionais Abertos (REAs) na perspectiva da Ciência da Informação: um estudo dos aspectos relacionados à produção e ao uso. 2018. 224 f. Dissertação (Mestrado em Ciência da Informação) - Universidade de São Paulo, São Paulo, 2018. Disponível em: https:/teses.usp.br/teses/disponiveis/27/27151/tde-13092018-145712/ptbr.php. Acesso em: 1 out. 2021.

\section{HIRST, T. Open Educational Resources and the University}

Library Website, 2019. Disponível em: https://blog.ouseful.info/2019/08/10/open-educational-resources-and-theuniversity-library-website/. Acesso em: 20 set. 2021.

KLEYMEER, P.; KLEINMAN, M.; HANSS, T. Reaching the heart of the university: libraries and the future of OER. In: ANNUAL OPEN EDUCATION CONFERENCE, 7., 2010, Barcelona. Eletronic procedings [...]. Barcelona: Open Education, 2010. Disponível em:

https://deepblue.lib.umich.edu/bitstream/handle/2027.42/78006/ReachingtheHe artoftheUniversity-KleymeerKleinmanHanss. pdf?sequence $=1$ \&isAllowed $=y$. Acesso em: 10 out. 2021.

LJUBLJANA OER ACTION PLAN. Ljubljana OER Action Plan 2017. Disponível em: https://en.unesco.org/sites/default/files/jubljana_oer_action_plan_2017.pdf. Acesso em: 20 mar. 2021.

LÜDKE, M.; ANDRÉ, M. E. D. A. Pesquisa em educação: abordagens qualitativas. São Paulo: EPU; 1986. 99 p.

MORAES, M. C. O paradigma educacional emergente. Campinas: Papirus, 1997.

ORGANIZAÇÃO DAS NAÇÕES UNIDAS PARA A EDUCAÇÃO, A CIÊNCIA E A CULTURA (UNESCO). OER Declaration. In: CONGRESSO MUNDIAL 
SOBRE RECURSOS EDUCACIONAIS ABERTOS (REA), 1., 2012. Paris: 2012. Disponível em:

http://www.unesco.org/new/fileadmin/MULTIMEDIA/HQ/CI/WPFD2009/Portugu ese_Declaration.html. Acesso em: 6 jun. 2021.

OPEN TEXTBOOK NETWORK. Open Textbooks Matter. Disponível em: https://open.umn.edu/opentextbooks/about. Acesso em: 10 nov. 2020.

PÖTTKER, L. M. V.; FERNEDA, E.; MOREIRO-GONZÁLEZ, J. A. Repositórios de objetos de aprendizagem: da arquitetura aos padrões de metadados para catalogação dos recursos educacionais. In: SEMINARIO EM CIÊNCIA DA INFORMAÇÃO, 6., 2016. Londrina: UEL, 2016. Disponível em: http://www.uel.br/eventos/cinf/index.php/secin2016/secin2016/paper/view/260/1 85. Acesso em: 5 jun. 2021.

PRUDENCIO, D. S.; BERNARDI, G.; BIOLCHINI, J. C. A. As contribuições dos recursos educacionais abertos para a promoção da competência em informação no campo da saúde. Revista Brasileira de Biblioteconomia e Documentação, [S.I.], v. 16, p. 1-28, 2020. Acesso em: 15 jun. 2021.

SANTANA, B.; ROSSINI, C.; DE LUCA PRETTO, N. Recursos Educacionais Abertos: práticas colaborativas e políticas públicas. Salvador: EDUFBA, 2012.

STACHOKAS, G. A new classification system for free electronic resources.

Serials Review, [S.I.], v. 38, p. 12-16, 2012. Disponível em: https://www.tandfonline.com/doi/abs/10.1080/00987913.2012.10765413. Acesso em: 5 abr. 2020.

\title{
OER AND LIBRARIES: DISCUSSIONS ON THE IMPORTANCE OF CLASSIFICATION AND REPRESENTATION
}

\begin{abstract}
Objective: Discuss the manifestations of open educational resources (oer) in libraries, present the speec method and the metadata standard ieee lom - learning object metadata. Methodology: research of an exploratory and descriptive nature that uses bibliographic research and a qualitative approach to present the results. Results: shows the importance of classification and descriptive representation processes for the search, access, use and dissemination of open educational resources. Conclusions: it concludes that the processes of organization, classification and representation of oer influence the recoverability of resources in virtual catalogs and, consequently, their promotion, access and use.
\end{abstract}

Descriptors: Open Educational Resource. Information Resource. Information Source. Learning Object Metadata. 


\title{
REA Y BIBLIOTECAS: DISCUSIONES SOBRE LA IMPORTANCIA DE LA CALIFICACIÓN Y REPRESENTACIÓN
}

\begin{abstract}
RESUMEN
Objetivo: Discutir las manifestaciones de los recursos educativos abiertos (REA) en las bibliotecas, presentar el método SPEEC y el estándar de metadatos IEEE LOM Learning Object Metadata. Metodología: Investigación de carácter exploratorio y descriptivo que utiliza la investigación bibliográfica y un enfoque cualitativo para presentar los resultados. Resultados: Presenta la importancia de los procesos de clasificación y representación descriptiva para la búsqueda, acceso, uso y difusión de recursos educativos abiertos. Conclusiones: Se concluye que los procesos de organización, clasificación y representación de los REA influyen en la recuperabilidad de los recursos en los catálogos virtuales y, en consecuencia, en su promoción, acceso y uso.
\end{abstract}

Descriptores: Recurso educativo abierto. Recurso de información. Fuente de información. Metadatos del objeto de aprendizaje.

Recebido em: 13.10 .2021

Aceito em: 27.12.2021 